ICIFSS, 8-10 January 2018, Erode, Tamil Nadu, India

Notes on Intuitionistic Fuzzy Sets

Print ISSN 1310-4926, Online ISSN 2367-8283

Vol. 24, 2018, No. 1, 1-12

DOI: 10.7546/nifs.2018.24.1.1-12

\title{
On interval-valued intuitionistic fuzzy modal operators
}

\author{
Krassimir T. Atanassov \\ Department of Bioinformatics and Mathematical Modelling \\ Institute of Biophysics and Biomedical Engineering \\ Bulgarian Academy of Sciences \\ Acad. G. Bonchev Str., B1. 105, Sofia-1113, Bulgaria, \\ and \\ Intelligent Systems Laboratory, Prof. Dr. Asen Zlatarov University \\ 1 Yakim Yakimov Blvd., Burgas-8010, Bulgaria \\ e-mail: kratebas.bg
}

Received: 1 October 2017

Accepted: 27 October 2017

Abstract: An survey of the existing interval-valued intuitionistic fuzzy modal operators is given. Eight new operators are introduced that extend the older ones. Some of their basic properties are discussed. Open problems are formulated.

Keywords: Interval-valued intuitionistic fuzzy set, Interval-valued intuitionistic fuzzy operator 2010 Mathematics Subject Classification: 03E72.

\section{Introduction}

An Interval-Valued Intuitionistic Fuzzy Set (IVIFS) $A^{*}$ (over a basic set $E$ ) is an object of the form: $A^{*}=\left\{\left\langle x, M_{A}(x), N_{A}(x)\right\rangle \mid x \in E\right\}$, where $A \subseteq E, M_{A}(x) \subset[0,1]$ and $N_{A}(x) \subset[0,1]$ are intervals and for all $x \in E$ :

$$
\sup M_{A}(x)+\sup N_{A}(x) \leq 1 .
$$

This definition is analogous to the definition of an IFS, that is a partial case of an IVIFS for the case, when $\mu_{A}(x)=\inf M_{A}(x)=\sup M_{A}(x), \mu_{A}(x)=\inf N_{A}(x)=\sup N_{A}(x)$, and

$$
\mu_{A}(x)+\nu_{A}(x)=\sup M_{A}(x)+\sup N_{A}(x) \leq 1 .
$$


The definition of the IVIFS can be however rewritten to become an analogue of the second definition of the IFS (see [5]) - namely, if $M_{A}$ and $N_{A}$ are interpreted as functions. Then, an IVIFS $A$ (over a basic set $E$ ) is given by functions

$$
M_{A}: E \rightarrow I N T([0,1]) \text { and } N_{A}: E \rightarrow I N T([0,1])
$$

and the above inequality.

We must note that there is no difference in principle between the two approaches. And what is more, the same exist also in the ordinary fuzzy sets theory. The author originally used the first one influenced by the Kaufmann's book [11]. Perhaps it was this approach that helped him develop the theory of operators over IFS in its present form. The same notation was used in 1987-1988 in the research on IVIFSs, too (see $[3,10]$ ).

Because below we will use only notation $A^{*}$, for brevity, the asterisk will be omitted.

IVIFSs have geometrical interpretations similar to, but more complex than these of the IFSs (Fig. 1).

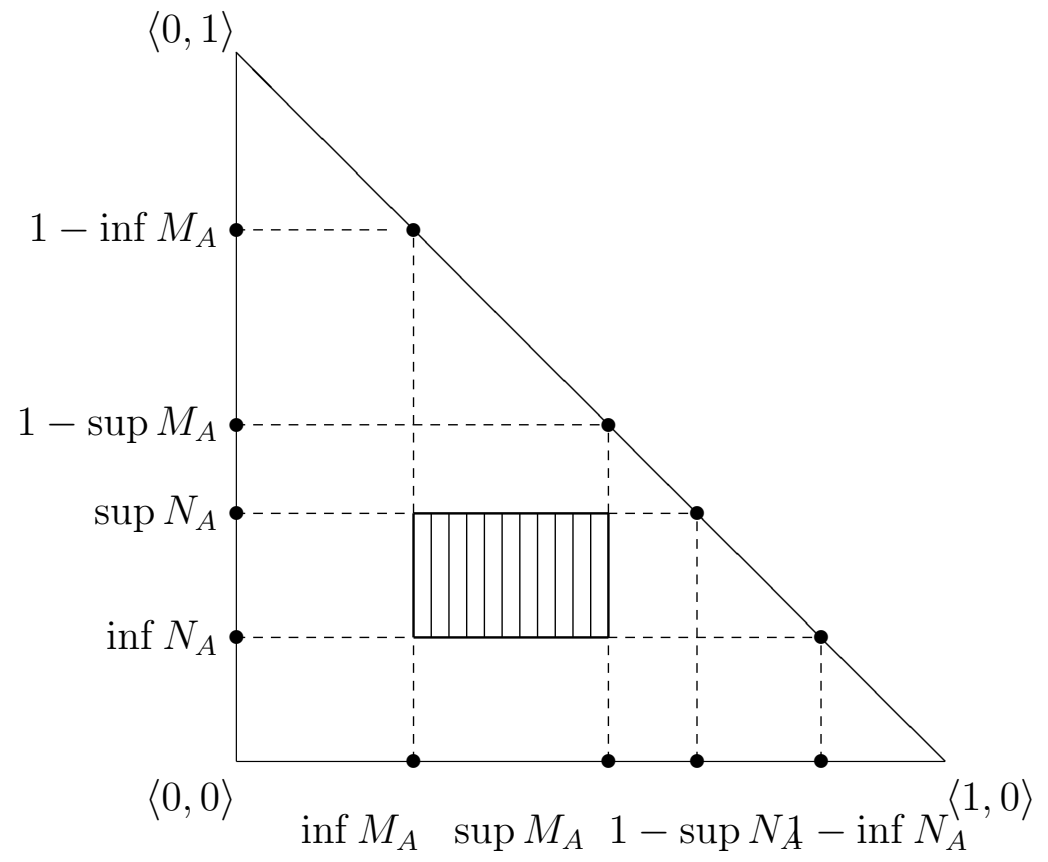

Figure 1.

It is suitable to define $P_{A}(x)=\left[0,1-\sup M_{A}(x)-\sup N_{A}(x)\right]$.

Therefore, $\inf P_{A}(x)=0$ and

$$
\sup P_{A}(x)=1-\sup M_{A}(x)-\sup N_{A}(x) .
$$

First, we define some relations over IVFSs. For every two IVIFSs $A$ and $B$ the following relations hold ("iff", is a abbriviation of "if and only if"):

$$
\begin{array}{lll}
A \subset_{\square, \text { inf }} B \quad \text { iff }^{1} & (\forall x \in E)\left(\inf M_{A}(x) \leq \inf M_{B}(x)\right), \\
A \subset_{\square, \text { sup }} B \quad \text { iff } & (\forall x \in E)\left(\sup M_{A}(x) \leq \sup M_{B}(x)\right), \\
A \subset_{\diamond, \text { inf }} B \quad \text { iff } & (\forall x \in E)\left(\inf N_{A}(x) \geq \inf N_{B}(x)\right), \\
A \subset_{\diamond, \sup } B \quad \text { iff } & (\forall x \in E)\left(\sup N_{A}(x) \geq \sup N_{B}(x)\right),
\end{array}
$$




$$
\begin{array}{lll}
A \subset_{\square} B & \text { iff } & A \subset_{\square, \text { inf }} B \& A \subset_{\square, \text { sup }} B, \\
A \subset_{\diamond} B & \text { iff } & A \subset_{\diamond, \text { inf }} B \& A \subset_{\diamond \text {,sup }} B, \\
A \subset B & \text { iff } & A \subset_{\square} B \& B \subset_{\diamond} A, \\
A \subseteq_{\square, \text { inf }} B & \text { iff } & (\forall x \in E)\left(\inf M_{A}(x) \leq \inf M_{B}(x)\right), \\
A \subseteq_{\square, \text { sup }} B & \text { iff } & (\forall x \in E)\left(\sup M_{A}(x) \leq \sup M_{B}(x)\right), \\
A \subseteq_{\diamond, \text { inf }} B & \text { iff } & (\forall x \in E)\left(\inf N_{A}(x) \geq \inf N_{B}(x)\right), \\
A \subseteq_{\diamond, \text { sup }} B & \text { iff } & (\forall x \in E)\left(\sup N_{A}(x) \geq \sup N_{B}(x)\right), \\
A \subseteq_{\square} B & \text { iff } & A \subseteq_{\square, \text { inf }} B \& A \subseteq_{\square, \text { sup }} B, \\
A \subseteq_{\diamond} B & \text { iff } & A \subseteq_{\diamond, \text { inf }} B \& A \subseteq_{\diamond, \text { sup }} B, \\
A \subseteq B & \text { iff } & A \subseteq_{\square} B \& B \subseteq_{\diamond} A, \\
A=B & \text { iff } & A \subset B \& B \subset A,
\end{array}
$$

Second, we describe the basic operations, defined for every two IVIFSs $A$ and $B$. They are:

$$
\begin{aligned}
\neg A= & \left\{\left\langle x, N_{A}(x), M_{A}(x)\right\rangle \mid x \in E\right\}, \\
A \cap B= & \left\{\left\langlex,\left[\min \left(\inf M_{A}(x), \inf M_{B}(x)\right), \min \left(\sup M_{A}(x), \sup M_{B}(x)\right)\right],\right.\right. \\
& {\left.\left.\left[\max \left(\inf N_{A}(x), \inf N_{B}(x)\right), \max \left(\sup N_{A}(x), \sup N_{B}(x)\right)\right]\right\rangle \mid x \in E\right\}, } \\
A \cup B= & \left\{\left\langlex,\left[\max \left(\inf M_{A}(x), \inf M_{B}(x)\right), \max \left(\sup M_{A}(x) \sup M_{B}(x)\right)\right],\right.\right. \\
& {\left.\left.\left[\min \left(\inf N_{A}(x), \inf N_{B}(x)\right), \min \left(\sup N_{A}(x), \sup N_{B}(x)\right)\right]\right\rangle \mid x \in E\right\} } \\
A+B= & \left\{\left\langlex,\left[\inf M_{A}(x)+\inf M_{B}(x)-\inf M_{A}(x) \cdot \inf M_{B}(x),\right.\right.\right. \\
& \left.\sup M_{A}(x)+\sup M_{B}(x)-\sup M_{A}(x) \cdot \sup M_{B}(x)\right], \\
& {\left.\left.\left[\inf N_{A}(x) \cdot \inf N_{B}(x), \sup N_{A}(x) \cdot \sup N_{B}(x)\right]\right\rangle \mid x \in E\right\} } \\
= & \left\{\left\langlex,\left[\inf M_{A}(x) \cdot \inf M_{B}(x), \sup M_{A}(x) \cdot \sup M_{B}(x)\right],\right.\right. \\
& {\left[\inf N_{A}(x)+\inf N_{B}(x)-\inf N_{A}(x) \cdot \inf N_{B}(x),\right.} \\
& \left.\left.\left.\sup N_{A}(x)+\sup N_{B}(x)-\sup N_{A}(x) \cdot \sup N_{B}(x)\right]\right\rangle \mid x \in E\right\} \\
A . B= & \left\{\left\langlex,\left[\left(\inf M_{A}(x)+\inf M_{B}(x)\right) / 2,\left(\sup M_{A}(x)+\sup M_{B}(x)\right) / 2\right],\right.\right. \\
& {\left.\left.\left[\left(\inf N_{A}(x)+\inf N_{B}(x)\right) / 2,\left(\sup N_{A}(x)\right\rangle+\sup N_{B}(x)\right) / 2\right] \mid x \in E\right\} }
\end{aligned}
$$

Now, over IFSs 185 different implications are defined (see, e.g., [6, 7]). On their basis, three types of 185 conjunctions and discunctions can be introduced (see, e.g., [1,2]. After publishing of [7], 5 new implications were introduced, but for them there are not constructed new conjunctions and discunctions. Now, the following Open Problems are interesting: 1. To construct analogous of all 190 implicationss for the IVIFS-case; 2 . On their basis, to construct the respective triples of conjunctions and discunctions.

Third, we give the list of the operators of modal type that are defined over an IVIFS $A$ (see 
also $[4,5])$ :

$$
\begin{aligned}
\square A= & \left\{\left\langle x, M_{A}(x),\left[\inf N_{A}(x), 1-\sup M_{A}(x)\right]\right\rangle \mid x \in E\right\}, \\
\diamond A= & \left\{\left\langle x,\left[\inf M_{A}(x), 1-\sup N_{A}(x)\right], N_{A}(x)\right\rangle \mid x \in E\right\}, \\
D_{\alpha}(A)= & \left\{\left\langlex,\left[\inf M_{A}(x), \sup M_{A}(x)+\alpha \cdot\left(1-\sup M_{A}(x)-\sup N_{A}(x)\right)\right],\right.\right. \\
& {\left.\left.\left[\inf N_{A}(x), \sup N_{A}(x)+(1-\alpha) \cdot\left(1-\sup M_{A}(x)-\sup N_{A}(x)\right)\right]\right\rangle \mid x \in E\right\}, } \\
F_{\alpha, \beta}(A)= & \left\{\left\langlex,\left[\inf M_{A}(x), \sup M_{A}(x)+\alpha \cdot\left(1-\sup M_{A}(x)-\sup N_{A}(x)\right)\right],\right.\right. \\
& {\left.\left.\left[\inf N_{A}(x), \sup N_{A}(x)+\beta \cdot\left(1-\sup M_{A}(x)-\sup N_{A}(x)\right)\right]\right\rangle \mid x \in E\right\}, \alpha+\beta \leq 1, } \\
G_{\alpha, \beta}(A)= & \left\{\left\langle x,\left[\alpha \cdot \inf M_{A}(x), \alpha \cdot \sup M_{A}(x)\right],\left[\beta \cdot \inf N_{A}(x), \beta \cdot \sup N_{A}(x)\right]\right\rangle \mid x \in E\right\}, \\
H_{\alpha, \beta}(A)= & \left\{\left\langlex,\left[\alpha \cdot \inf M(x), \alpha \cdot \sup M_{A}(x)\right],\left[\inf N_{A}(x), \sup N_{A}(x)\right.\right.\right. \\
& \left.\left.\left.+\beta \cdot\left(1-\sup M_{A}(x)-\sup N_{A}(x)\right)\right]\right\rangle \mid x \in E\right\}, \\
H_{\alpha, \beta}^{*}(A)= & \left\{\left\langlex,\left[\alpha \cdot \inf M_{A}(x), \alpha \cdot \sup M_{A}(x)\right],\left[\inf N_{A}(x), \sup N_{A}(x)\right.\right.\right. \\
& \left.\left.\left.+\beta \cdot\left(1-\alpha \cdot \sup M_{A}(x)-\sup N_{A}(x)\right)\right]\right\rangle \mid x \in E\right\}, \\
J_{\alpha, \beta}(A)= & \left\{\left\langlex,\left[\inf M_{A}(x), \sup M_{A}(x)+\alpha \cdot\left(1-\sup M_{A}(x)\right.\right.\right.\right. \\
& \left.\left.\left.\left.-\sup N_{A}(x)\right)\right],\left[\beta \cdot \inf N_{A}(x), \beta \cdot \sup N_{A}(x)\right]\right\rangle \mid x \in E\right\}, \\
J_{\alpha, \beta}^{*}(A)= & \left\{\left\langlex,\left[\inf M_{A}(x), \sup M_{A}(x)+\alpha \cdot\left(1-\sup M_{A}(x)\right.\right.\right.\right. \\
& \left.\left.\left.\left.-\beta \cdot \sup N_{A}(x)\right)\right],\left[\beta \cdot \inf N_{A}(x), \beta \cdot \sup N_{A}(x)\right]\right\rangle \mid x \in E\right\},
\end{aligned}
$$

where $\alpha, \beta \in[0,1]$.

Obviously, $\square A=D_{0}(A)=F_{0,1}(A), \diamond A=D_{1}(A)=F_{1,0}(A), D_{\alpha}(A)=F_{\alpha, 1-\alpha}(A)$ and by this reason, below we will not discuss these three operators.

In [5], the operators $F_{\alpha . \beta}, \ldots, J_{\alpha, \beta}^{*}$ are extended to the following operators, where $\alpha, \beta, \gamma, \delta \in$ $[0,1]$ such that $\alpha \leq \beta$ and $\gamma \leq \delta$ :

$$
\begin{aligned}
\bar{F}_{\alpha, \beta, \gamma, \delta}(A)= & \left\{\left\langlex,\left[\inf M_{A}(x)+\alpha \cdot\left(1-\sup M_{A}(x)-\sup N_{A}(x)\right),\right.\right.\right. \\
& \left.\sup M_{A}(x)+\beta \cdot\left(1-\sup M_{A}(x)-\sup N_{A}(x)\right)\right], \\
& {\left[\inf N_{A}(x)+\gamma \cdot\left(1-\sup M_{A}(x)-\sup N_{A}(x)\right),\right.} \\
& \left.\left.\left.\sup N_{A}(x)+\delta \cdot\left(1-\sup M_{A}(x)-\sup N_{A}(x)\right)\right]\right\rangle \mid x \in E\right\}, \beta+\delta \leq 1, \\
& \left\{\left\langlex,\left[\alpha \cdot \inf M_{A}(x), \beta \cdot \sup M_{A}(x)\right],\right.\right. \\
& {\left.\left.\left[\gamma \cdot \inf N_{A}(x), \delta \cdot \sup N_{A}(x)\right]\right\rangle \mid x \in E\right\}, } \\
\bar{G}_{\alpha, \beta, \gamma, \delta}(A)= & \left\{\left\langlex,\left[\alpha \cdot \inf M_{A}(x), \beta \cdot \sup M_{A}(x)\right],\right.\right. \\
\bar{H}_{\alpha, \beta, \gamma, \delta}(A)=\operatorname{sinf} N_{A}(x)+\gamma \cdot\left(1-\sup M_{A}(x)-\sup N_{A}(x)\right), & \left.\left.\left.\sup N_{A}(x)+\delta \cdot\left(1-\sup M_{A}(x)-\sup N_{A}(x)\right)\right]\right\rangle \mid x \in E\right\} \\
& \left\{\left\langlex,\left[\alpha \cdot \inf M_{A}(x), \beta \cdot \sup M_{A}(x)\right],\right.\right. \\
& {\left[\inf N_{A}(x)+\gamma \cdot\left(1-\beta \cdot \sup M_{A}(x)-\sup N_{A}(x)\right),\right.} \\
& \left.\left.\left.\sup N_{A}(x)+\delta \cdot\left(1-\beta \cdot \sup M_{A}(x)-\sup N_{A}(x)\right)\right]\right\rangle \mid x \in E\right\}
\end{aligned}
$$




$$
\begin{aligned}
\bar{J}_{\alpha, \beta, \gamma, \delta}(A)= & \left\{\left\langlex,\left[\inf M_{A}(x)+\alpha \cdot\left(1-\sup M_{A}(x)-\sup N_{A}(x)\right),\right.\right.\right. \\
& \left.\sup M_{A}(x)+\beta \cdot\left(1-\sup M_{A}(x)-\sup N_{A}(x)\right)\right], \\
& {\left.\left.\left[\gamma \cdot \inf N_{A}(x), \delta \cdot \sup N_{A}(x)\right]\right\rangle \mid x \in E\right\} } \\
\bar{J}_{\alpha, \beta, \gamma, \delta}^{*}(A)= & \left\{\left\langlex,\left[\inf M_{A}(x)+\alpha \cdot\left(1-\delta \cdot \sup M_{A}(x)-\sup N_{A}(x)\right),\right.\right.\right. \\
& \left.\sup M_{A}(x)+\beta \cdot\left(1-\sup M_{A}(x)-\delta \cdot \sup N_{A}(x)\right)\right], \\
& {\left.\left.\left[\gamma \cdot \inf N_{A}(x), \delta \cdot \sup N_{A}(x)\right]\right\rangle \mid x \in E\right\} . }
\end{aligned}
$$

Obviosly, the new operators are extensions of the previous ones.

In $[6,8]$, the idea for changing of real number parameters $\alpha, \beta$ in the operators defined over IFS $A$ with whole IFS $B$ is discussed. Here, we discuss wimilar idea for the case, when set $A$ is an IVIFS.

\section{Main results}

Here, following [8], we describe step by step 8 intuitionistic fuzzy extended modal operators and discuss some of their propertiers. We give the proof of only the first two properties, formulated below and the rest properties are proved by the same manner.

\subsection{Operator $F_{B}$}

Let $B$ be an IVIFS. Then for each IVIFS $A$ :

$$
\begin{gathered}
F_{B}(A)=\left\{\left\langlex,\left[\inf M_{A}(x)+\inf M_{B}(x) \sup P_{A}(x), \sup M_{A}(x)+\sup M_{B}(x) \sup P_{A}(x)\right],\right.\right. \\
\left.\left.\left[\inf N_{A}(x)+\inf N_{B}(x) \sup P_{A}(x), \sup N_{A}(x)+\sup N_{B}(x) \sup P_{A}(x)\right]\right\rangle \mid x \in E\right\} .
\end{gathered}
$$

Theorem 1. For every six IFSs $A, B, C, D, P, Q$ :

$$
\begin{gathered}
\neg F_{\neg B}(\neg A)=F_{B}(A), \\
F_{B}\left(F_{C}(A)\right)=F_{F_{B}(C)}(A), \\
F_{B}(A \cap D) \subseteq F_{B}(A) \cap F_{B}(D), \\
F_{B}(A \cup D) \supseteq F_{B}(A) \cup F_{B}(D), \\
F_{B}(A @ D)=F_{B}(A) @ F_{B}(D), \\
F_{B}(A) \subseteq F_{C}(A), \quad \text { where } B \subseteq C .
\end{gathered}
$$

Proof: Let the IVIFSs $A$ and $B$ be given. Then, for the first equality we obtain that

$$
\begin{gathered}
\neg F_{\neg B}(\neg A)=\neg F_{\neg\left\{\left\langle x, M_{B}(x), N_{B}(x)\right\rangle \mid x \in E\right\}}(\neg A) \\
=\neg F_{\left\{\left\langle x, N_{B}(x), M_{B}(x)\right\rangle \mid x \in E\right\}}\left(\left\{\left\langle x, N_{A}(x), M_{A}(x)\right\rangle \mid x \in E\right\}\right) \\
=\neg\left\{\left\langlex,\left[\inf N_{A}(x)+\inf N_{B}(x) \sup P_{A}(x), \sup N_{A}(x)+\sup N_{B}(x) \sup P_{A}(x)\right],\right.\right. \\
\left.\left.\left[\inf M_{A}(x)+\inf M_{B}(x) \sup P_{A}(x), \sup M_{A}(x)+\sup M_{B}(x) \sup P_{A}(x)\right]\right\rangle \mid x \in E\right\}
\end{gathered}
$$


$=\left\{\left\langle x,\left[\inf M_{A}(x)+\inf M_{B}(x) \sup P_{A}(x), \sup M_{A}(x)+\sup M_{B}(x) \sup P_{A}(x)\right]\right.\right.$, $\left.\left.\left[\inf N_{A}(x)+\inf N_{B}(x) \sup P_{A}(x), \sup N_{A}(x)+\sup N_{B}(x) \sup P_{A}(x)\right]\right\rangle \mid x \in E\right\}$

$$
=F_{B}(A) \text {. }
$$

For the second equality we obtain

$$
F_{B}\left(F_{C}(A)\right)
$$

$=F_{B}\left(\left\{\left\langle x,\left[\inf M_{A}(x)+\inf M_{C}(x) \sup P_{A}(x), \sup M_{A}(x)+\sup M_{C}(x) \sup P_{A}(x)\right]\right.\right.\right.$, $\left.\left.\left.\left[\inf N_{A}(x)+\inf N_{C}(x) \sup P_{A}(x), \sup N_{A}(x)+\sup N_{C}(x) \sup P_{A}(x)\right]\right\rangle \mid x \in E\right\}\right)$

$=\left\{\left\langle x,\left[\inf M_{A}(x)+\inf M_{C}(x) \sup P_{A}(x)+\inf M_{B}(x)\left(1-\sup M_{A}(x)\right.\right.\right.\right.$

$\left.-\sup M_{C}(x) \sup P_{A}(x)-\sup N_{A}(x)-\sup N_{C}(x) \sup P_{A}(x)\right)$,

$\sup M_{A}(x)+\sup M_{C}(x) \sup P_{A}(x)+\sup M_{B}(x)\left(1-\sup M_{A}(x)\right.$

$\left.\left.-\sup M_{C}(x) \sup P_{A}(x)-\sup N_{A}(x)-\sup N_{C}(x) \sup P_{A}(x)\right)\right]$,

$\left[\inf N_{A}(x)+\inf N_{C}(x) \sup P_{A}(x)+\inf N_{B}(x)\left(1-\sup M_{A}(x)\right.\right.$

$\left.-\sup M_{C}(x) \sup P_{A}(x)-\sup N_{A}(x)-\sup N_{C}(x) \sup P_{A}(x)\right)$,

$\sup N_{A}(x)+\sup N_{C}(x) \sup P_{A}(x)+\sup N_{B}(x)\left(1-\sup M_{A}(x)\right.$

$\left.\left.\left.\left.-\sup M_{C}(x) \sup P_{A}(x)-\sup N_{A}(x)-\sup N_{C}(x) \sup P_{A}(x)\right)\right]\right\rangle \mid x \in E\right\}$

$=\left\{\left\langle x,\left[\inf M_{A}(x)+\inf M_{C}(x) \sup P_{A}(x)+\inf M_{B}(x)-\inf M_{B}(x) \sup M_{A}(x)\right.\right.\right.$

$\left.-\inf M_{B}(x) \sup M_{C}(x) \sup P_{A}(x)-\inf M_{B}(x) \sup N_{A}(x)-\inf M_{B}(x) \sup N_{C}(x) \sup P_{A}(x)\right)$,

$\sup M_{A}(x)+\sup M_{C}(x) \sup P_{A}(x)+\sup M_{B}(x)-\sup M_{B}(x) \sup M_{A}(x)$

$\left.\left.-\sup M_{B}(x) \sup M_{C}(x) \sup P_{A}(x)-\sup M_{B}(x) \sup N_{A}(x)-\sup M_{B}(x) \sup N_{C}(x) \sup P_{A}(x)\right)\right]$,

$\left[\inf N_{A}(x)+\inf N_{C}(x) \sup P_{A}(x)+\inf N_{B}(x)-\inf N_{B}(x) \sup M_{A}(x)\right.$

$\left.-\inf N_{B}(x) \sup M_{C}(x) \sup P_{A}(x)-\inf N_{B}(x) \sup N_{A}(x)-\inf N_{B}(x) \sup N_{C}(x) \sup P_{A}(x)\right)$,

$\sup N_{A}(x)+\sup N_{C}(x) \sup P_{A}(x)+\sup N_{B}(x)-\sup N_{B}(x) \sup M_{A}(x)$

$-\sup N_{B}(x) \sup M_{C}(x) \sup P_{A}(x)-\sup N_{B}(x) \sup N_{A}(x)$

$\left.\left.\left.\left.-\sup N_{B}(x) \sup N_{C}(x) \sup P_{A}(x)\right)\right]\right\rangle \mid x \in E\right\}$

$=\left\{\left\langle x,\left[\inf M_{A}(x)+\inf M_{B}(x)\left(1-\sup M_{A}(x)-\sup N_{A}(x)\right)\right.\right.\right.$

$\left.+\left(\inf M_{C}(x)-\inf M_{B}(x) \sup M_{C}(x)-\inf M_{B}(x) \sup N_{C}(x)\right) \sup P_{A}(x)\right)$,

$\sup M_{A}(x)+\sup M_{B}(x)\left(1-\sup M_{A}(x)-\sup N_{A}(x)\right)$

$\left.\left.+\left(\sup M_{C}(x)-\sup M_{B}(x) \sup M_{C}(x)-\sup M_{B}(x) \sup N_{C}(x)\right) \sup P_{A}(x)\right)\right]$,

$\left[\inf N_{A}(x)+\inf N_{B}(x)\left(1-\sup M_{A}(x)-\sup N_{A}(x)\right)\right.$ 


$$
\begin{gathered}
\left.+\left(\inf N_{C}(x)-\inf N_{B}(x) \sup M_{C}(x)-\inf N_{B}(x) \sup N_{C}(x)\right) \sup P_{A}(x)\right), \\
\sup N_{A}(x)+\sup N_{B}(x)\left(1-\sup M_{A}(x)-\sup N_{A}(x)\right) \\
\left.\left.\left.\left.+\left(\sup N_{C}(x)-\sup N_{B}(x) \sup M_{C}(x)-\sup N_{B}(x) \sup N_{C}(x)\right) \sup P_{A}(x)\right)\right]\right\rangle \mid x \in E\right\}
\end{gathered}
$$

(from $(*)$ )

$$
\begin{gathered}
=\left\{\left\langlex,\left[\inf M_{A}(x)+\inf M_{B}(x) \sup P_{A}(x)\right.\right.\right. \\
\left.+\left(\inf M_{C}(x)-\inf M_{B}(x) \sup M_{C}(x)-\inf M_{B}(x) \sup N_{C}(x)\right) \sup P_{A}(x)\right), \\
\sup M_{A}(x)+\sup M_{B}(x) \sup P_{A}(x) \\
\left.\left.+\left(\sup M_{C}(x)-\sup M_{B}(x) \sup M_{C}(x)-\sup M_{B}(x) \sup N_{C}(x)\right) \sup P_{A}(x)\right)\right], \\
{\left[\inf N_{A}(x)+\inf N_{B}(x) \sup P_{A}(x)\right.} \\
\left.+\left(\inf N_{C}(x)-\inf N_{B}(x) \sup M_{C}(x)-\inf N_{B}(x) \sup N_{C}(x)\right) \sup P_{A}(x)\right), \\
\sup N_{A}(x)+\sup N_{B}(x) \sup P_{A}(x) \\
\left.\left.\left.\left.+\left(\sup N_{C}(x)-\sup N_{B}(x) \sup M_{C}(x)-\sup N_{B}(x) \sup N_{C}(x)\right) \sup P_{A}(x)\right)\right]\right\rangle \mid x \in E\right\} \\
=\left\{\left\langlex,\left[\inf M_{A}(x)+\left(\inf M_{B}(x)+\inf M_{C}(x)-\inf M_{B}(x) \sup M_{C}(x)\right.\right.\right.\right. \\
\left.\left.-\inf M_{B}(x) \sup N_{C}(x)\right) \sup P_{A}(x)\right), \\
\sup M_{A}(x)+\left(\sup M_{B}(x)+\sup M_{C}(x)-\sup M_{B}(x) \sup M_{C}(x)\right. \\
\left.\left.\left.-\sup M_{B}(x) \sup N_{C}(x)\right) \sup P_{A}(x)\right)\right], \\
{\left[\inf N_{A}(x)+\left(\inf N_{B}(x)+\inf N_{C}(x)-\inf N_{B}(x) \sup M_{C}(x)\right.\right.} \\
\left.\left.-\inf N_{B}(x) \sup N_{C}(x)\right) \sup P_{A}(x)\right), \\
\left.\left.\left.\left.\left.-\sup N_{B}(x) \sup N_{C}(x)\right) \sup P_{A}(x)\right)\right]\right\rangle \mid x \in E\right\} \\
\sup N_{A}(x)+\left(\sup N_{B}(x)+\sup N_{C}(x)-\sup N_{B}(x) \sup M_{C}(x)\right.
\end{gathered}
$$

(let us denote conditionaly this set as)

$$
=F_{X}(A)
$$

where

$$
\begin{gathered}
X=\left\{\left\langle x, M_{X}(x), N_{X}(x)\right\rangle \mid x \in E\right\}, \\
\inf M_{X}(x)=\inf M_{B}(x)+\inf M_{C}(x)-\inf M_{B}(x) \sup M_{C}(x)-\inf M_{B}(x) \sup N_{C}(x), \\
\sup M_{X}(x)=\sup M_{B}(x)+\sup M_{C}(x)-\sup M_{B}(x) \sup M_{C}(x)-\sup M_{B}(x) \sup N_{C}(x), \\
\inf N_{X}(x)=\inf N_{B}(x)+\inf N_{C}(x)-\inf N_{B}(x) \sup M_{C}(x)-\inf N_{B}(x) \sup N_{C}(x), \\
\sup N_{X}(x)=\sup N_{B}(x)+\sup N_{C}(x)-\sup N_{B}(x) \sup M_{C}(x)-\sup N_{B}(x) \sup N_{C}(x) .
\end{gathered}
$$

Now, we must prove that set $X$ is an IVIFS.

First, we see diretly, that

$$
\begin{gathered}
0 \leq \inf M_{B}(x) \sup P_{C}(x)+\inf M_{C}(x) \\
=\inf M_{B}(x)+\inf M_{C}(x)-\inf M_{B}(x) \sup M_{C}(x)-\inf M_{B}(x) \sup N_{C}(x)
\end{gathered}
$$




$$
\begin{gathered}
=\inf M_{X}(x) \leq \sup M_{X}(x) \\
=\sup M_{B}(x)+\sup M_{C}(x)-\sup M_{B}(x) \sup M_{C}(x)-\sup M_{B}(x) \sup N_{C}(x) \\
=\sup M_{B}(x)\left(1-\sup M_{C}(x)-\sup N_{C}(x)\right)+\sup M_{C}(x) \\
=\sup M_{B}(x) \sup P_{C}(x)+\sup M_{C}(x) \\
\leq \sup P_{C}(x)+\sup M_{C}(x) \leq 1 .
\end{gathered}
$$

Second, analogously, we see that

$$
0 \leq \inf N_{X}(x) \leq \sup N_{X}(x) \leq 1
$$

Third, we check validity of condition (*) for set $X$.

$$
\begin{gathered}
\sup M_{X}(x)+\sup N_{X}(x) \\
=\sup M_{B}(x)+\sup M_{C}(x)-\sup M_{B}(x) \sup M_{C}(x)-\sup M_{B}(x) \sup N_{C}(x) \\
+\sup N_{B}(x)+\sup N_{C}(x)-\sup N_{B}(x) \sup M_{C}(x)-\sup N_{B}(x) \sup N_{C}(x) \\
=\sup M_{B}(x)\left(1-\sup M_{C}(x)-\sup N_{C}(x)\right)+\sup M_{C}(x) \\
+\sup N_{B}(x)\left(1-\sup M_{C}(x)-\sup N_{C}(x)\right)+\sup N_{C}(x)
\end{gathered}
$$

$($ from $(*))$

$$
\begin{gathered}
=\sup M_{B}(x) \sup P_{C}(x)+\sup N_{B}(x) \sup P_{C}(x) \\
=\left(\sup M_{B}(x)+\sup N_{B}(x)\right) \sup P_{C}(x) \leq \sup P_{C}(x) \leq 1 .
\end{gathered}
$$

Therefore, set $X$ is an IVIFS.

Finally, se wee that

$$
\begin{gathered}
\inf M_{X}(x)=\inf M_{C}(x)+\inf M_{B}(x)\left(1-\sup M_{C}(x)-\sup N_{C}(x)\right) \\
=\inf M_{C}(x)+\inf M_{B}(x) \sup P_{C}(x), \\
\sup M_{X}(x)=\sup M_{C}(x)+\sup M_{B}(x)\left(1-\sup M_{C}(x)-\sup N_{C}(x)\right) \\
=\sup M_{C}(x)+\sup M_{B}(x) \sup P_{C}(x), \\
\inf N_{X}(x)=\inf N_{C}(x)+\inf N_{B}(x)\left(1-\sup M_{C}(x)-\sup N_{C}(x)\right) \\
=\inf N_{C}(x)+\inf N_{B}(x) \sup P_{C}(x), \\
\sup N_{X}(x)=\sup N_{C}(x)+\sup N_{B}(x)\left(1-\sup M_{C}(x)-\sup N_{C}(x)\right) \\
=\sup N_{C}(x)+\sup N_{B}(x) \sup M_{C}(x) .
\end{gathered}
$$

Therefore, $X=F_{B}(C)$. 


\subsection{Operator $G_{B, C}$}

Let $B$ and $C$ be IVIFSs. Then for each IVIFS $A$ :

$$
\begin{gathered}
G_{B, C}(A)=\left\{\left\langlex,\left[\inf M_{B}(x) \inf M_{A}(x), \sup M_{B}(x) \sup M_{A}(x)\right],\right.\right. \\
\left.\left.\left[\inf N_{C}(x) \inf N_{A}(x), \sup N_{C}(x) \sup N_{A}(x)\right]\right\rangle \mid x \in E\right\} .
\end{gathered}
$$

Theorem 2. For every six IFSs $A, B, C, D, P, Q$ :

$$
\begin{gathered}
G_{\square B, \diamond C}(A)=G_{B, C}(A) . \\
\neg G_{\neg C, \neg B}(\neg A)=G_{B, C}(A), \\
G_{B, C}\left(G_{P, Q}(A)\right)=G_{P, Q}\left(G_{B, C}(A)\right), \\
G_{B, C}(A \cap D)=G_{B, C}(A) \cap G_{B, C}(D), \\
G_{B, C}(A \cup D)=G_{B, C}(A) \cup G_{B, C}(D), \\
G_{B, C}(A) \subseteq G_{P, Q}(A), \text { where } B \subseteq P, C \subseteq Q .
\end{gathered}
$$

\subsection{Operator $H_{B, C}$}

It is defined for every three IVIFSs $A, B$ and $C$ by:

$$
\begin{gathered}
H_{B, C}(A)=\left\{\left\langlex,\left[\inf M_{B}(x) \inf M_{A}(x), \sup M_{B}(x) \sup M_{A}(x)\right],\right.\right. \\
\left.\left.\left[\inf N_{A}(x)+\inf N_{C}(x) \sup P_{A}(x), \sup N_{A}(x)+\sup N_{C}(x) \sup P_{A}(x)\right]\right\rangle \mid x \in E\right\} .
\end{gathered}
$$

Theorem 3. For every six IFSs $A, B, C, D, P, Q$, so that $B \subseteq P, C \subseteq Q$ :

$$
\begin{aligned}
& H_{\square B, \diamond C}(A)=H_{B, C}(A), \\
& \neg H_{\neg C, \neg B}(\neg A)=J_{B, C}(A),
\end{aligned}
$$

(see Subsection 2.6),

$$
\begin{gathered}
H_{B, C}(A \cap D) \subseteq H_{B, C}(A) \cap H_{B, C}(D), \\
H_{B, C}(A \cup D) \supseteq H_{B, C}(A) \cup H_{B, C}(D), \\
H_{B, C}(A) \subseteq H_{P, Q}(A), \quad \text { where } B \subseteq P, C \subseteq Q .
\end{gathered}
$$

\subsection{Operator $H_{B, C}^{*}$}

Let $B$ and $C$ be IVIFSs. Then for each IVIFS $A$ :

$$
\begin{gathered}
H_{B, C}^{*}(A)=\left\{\left\langlex,\left[\inf M_{B}(x) \inf M_{A}(x), \sup M_{B}(x) \sup M_{A}(x)\right],\right.\right. \\
{\left[\inf N_{A}(x)+\inf N_{C}(x)\left(1-\sup M_{B}(x) \sup M_{A}(x)-\sup N_{A}(x)\right),\right.} \\
\left.\left.\left.\sup N_{A}(x)+\sup N_{C}(x)\left(1-\sup M_{B}(x) \sup M_{A}(x)-\sup N_{A}(x)\right)\right]\right\rangle \mid x \in E\right\} .
\end{gathered}
$$


Theorem 4. For every three IFSs $A, B, C$ :

$$
\begin{aligned}
& H_{\square B, \diamond C}^{*}(A)=H_{B, C}^{*}(A), \\
& \neg H_{\neg C, \neg B}^{*}(\neg A)=J_{B, C}^{*}(A)
\end{aligned}
$$

(see Subsection 2.7),

$$
\begin{aligned}
& H_{B, C}^{*}(A \cap D) \subseteq H_{B, C}^{*}(A) \cap H_{B, C}(D), \\
& H_{B, C}^{*}(A \cup D) \supseteq H_{B, C}^{*}(A) \cup H_{B, C}(D) .
\end{aligned}
$$

\subsection{Operator $\bar{H}_{B, C}$}

It is defined for every three IVIFSs $A, B$ and $C$, so that

$$
\sup M_{B}(x)+\sup N_{C}(x) \leq 1
$$

for each $x \in E$, by:

$$
\begin{gathered}
\bar{H}_{B, C}(A)=\left\{\left\langlex,\left[\inf M_{B}(x) \inf M_{A}(x), \sup M_{B}(x) \sup M_{A}(x)\right],\right.\right. \\
{\left[\inf N_{A}(x)+\inf N_{C}(x)-\inf N_{A}(x) \inf N_{C}(x),\right.} \\
\left.\left.\left.\sup N_{A}(x)+\sup N_{C}(x)-\sup N_{A}(x) \sup N_{C}(x)\right]\right\rangle \mid x \in E\right\} .
\end{gathered}
$$

This operator is a modification of the operator $\bar{H}_{\alpha . \beta}$, defined in [9].

Theorem 5. For every five IFSs $A, B, C, P, Q$, so that $B \subseteq P, C \subseteq Q$ :

$$
\begin{aligned}
& \bar{H}_{\square B, \diamond C}(A)=\bar{H}_{B, C}(A), \\
& \neg \bar{H}_{\neg C, \neg B}(\neg A)=\bar{J}_{B, C}(A),
\end{aligned}
$$

(see Subsection 2.8),

$$
\begin{gathered}
\bar{H}_{B, C}(A \cap D) \subseteq \bar{H}_{B, C}(A) \cap \bar{H}_{B, C}(D), \\
\bar{H}_{B, C}(A \cup D) \supseteq \bar{H}_{B, C}(A) \cup \bar{H}_{B, C}(D), \\
\bar{H}_{B, C}(A) \subseteq \bar{H}_{P, Q}(A) .
\end{gathered}
$$

\subsection{Operator $J_{B, C}$}

Let $B$ and $C$ be IVIFSs. Then for each IVIFS $A$ the operator $J_{B, C}$ is defined by:

$$
\begin{gathered}
J_{B, C}(A)=\left\{\left\langlex,\left[\inf M_{A}(x)+\inf M_{B}(x) \sup P_{A}(x), \sup M_{A}(x)+\sup M_{B}(x) \sup P_{A}(x)\right],\right.\right. \\
\left.\left.\left[\inf N_{C}(x) \inf N_{A}(x), \sup N_{C}(x) \sup N_{A}(x)\right]\right\rangle \mid x \in E\right\} .
\end{gathered}
$$

Theorem 6. For every five IFSs $A, B, C, P, Q$, so that $B \subseteq P, C \subseteq Q$ :

$$
\begin{gathered}
J_{\square B, \diamond C}(A)=J_{B, C}(A), \\
\neg J_{\neg C, \neg B}(\neg A)=H_{B, C}(A), \\
J_{B, C}(A \cap D) \supseteq J_{B, C}(A) \cap J_{B, C}(D), \\
J_{B, C}(A \cup D) \subseteq J_{B, C}(A) \cup J_{B, C}(D), \\
J_{B, C}(A) \subseteq J_{P, Q}(A) .
\end{gathered}
$$




\subsection{Operator $J_{B, C}^{*}$}

Let $B$ and $C$ be IVIFSs. Then for each IVIFS $A$ :

$$
\begin{gathered}
J_{B, C}^{*}(A)=\left\{\left\langlex,\left[\inf M_{A}(x)+\inf M_{B}(x)\left(1-\sup M_{A}(x)-\sup N_{C}(x) \sup N_{A}(x)\right),\right.\right.\right. \\
\left.\sup M_{A}(x)+\sup M_{B}(x)\left(1-\sup M_{A}(x)-\sup N_{C}(x) \sup N_{A}(x)\right)\right], \\
\left.\left.\left[\inf N_{C}(x) \inf N_{A}(x), \sup N_{C}(x) \sup N_{A}(x)\right]\right\rangle \mid x \in E\right\} .
\end{gathered}
$$

Theorem 7. For every three IFSs $A, B, C$ :

$$
\begin{gathered}
J^{*}(A)=J_{B, C}^{*}(A), \\
\neg B, \diamond C \\
J_{\neg C, \neg B}^{*}(\neg A)=H_{B, C}^{*}(A), \\
J_{B, C}^{*}(A \cap D) \supseteq J_{B, C}^{*}(A) \cap J_{B, C}(D), \\
J_{B, C}^{*}(A \cup D) \subseteq J_{B, C}^{*}(A) \cup J_{B, C}(D) .
\end{gathered}
$$

\subsection{Operator $\bar{J}_{B, C}$}

It is defined for every three IVIFSs $A, B$ and $C$, so that $\sup M_{B}(x)+\sup N_{C}(x) \leq 1$ for each $x \in E$, by:

$$
\begin{gathered}
\bar{J}_{B, C}(A)=\left\{\left\langlex,\left[\inf M_{A}(x)+\inf M_{B}(x)-\inf M_{A}(x) \inf M_{B}(x),\right.\right.\right. \\
\left.\sup M_{A}(x)+\sup M_{B}(x)-\sup M_{A}(x) \sup M_{B}(x)\right], \\
\left.\left.\left[\inf N_{C}(x) \inf N_{A}(x), \sup N_{C}(x) \sup N_{A}(x)\right]\right\rangle \mid x \in E\right\} .
\end{gathered}
$$

This operator is a modification of the operator $\bar{J}_{\alpha . \beta}$, defined in [9].

Theorem 8. For every five IFSs $A, B, C, P, Q$, so that $B \subseteq P, C \subseteq Q$ :

$$
\begin{gathered}
\bar{J}_{\square B, \diamond C}(A)=\bar{J}_{B, C}(A), \\
\neg \bar{J}_{\neg C, \neg B}(\neg A)=\bar{H}_{B, C}(A), \\
\bar{J}_{B, C}(A \cap D) \supseteq \bar{J}_{B, C}(A) \cap \bar{J}_{B, C}(D), \\
\bar{J}_{B, C}(A \cup D) \subseteq \bar{J}_{B, C}(A) \cup \bar{J}_{B, C}(D), \\
\bar{J}_{B, C}(A) \subseteq \bar{J}_{P, Q}(A) .
\end{gathered}
$$

\section{Conclusion}

In a next research, other properties of the newly defined operators will be studied. All they can be used in a lot of areas of informatics and especially, in the Artificial Intelligence for modification of intuitionistic fuzzy evaluations.

Also, they can be used in Intercriteria Analysis procedures for changing of evaluations of the compared criteria.

An interesting Open Problem is: Can these operators be extended additionally? 


\section{Acknowledgements}

The author is thankful for the support provided by the Bulgarian National Science Fund under Grant Ref. No. DFNI-I-02-5 "InterCriteria Analysis: A New Approach to Decision Making”.

\section{References}

[1] Angelova, N., \& Stoenchev, M. (2016) Intuitionistic fuzzy conjunctions and disjunctions from first type. Annual of Informatics Section, Union of Scientists in Bulgaria, 8, 20152016, 1-17.

[2] Angelova, N., \& Stoenchev, M. (2017) Intuitionistic fuzzy conjunctions and disjunctions from third type. Notes on Intuitionistic Fuzzy Sets, 23(5), 29-41.

[3] Atanassov K. T. (1988) Review and New Results on Intuitionistic Fuzzy Sets, Mathematical Foundations of Artificial Intelligence Seminar, Sofia, 1988, Preprint IM-MFAIS-1-88. Reprinted: Int. J. Bioautomation, 2016, 20(S1), S7-S16.

[4] Atanassov K. (1994) Operators over interval-valued intuitionistic fuzzy sets, Fuzzy Sets and Systems, 64(2), 159-174.

[5] Atanassov, K. (1999) Intuitionistic Fuzzy Sets: Theory and Applications, Springer, Heidelberg.

[6] Atanassov, K. (2012) On Intuitionistic Fuzzy Sets Theory. Springer, Berlin.

[7] Atanassov, K. (2017) Intuitionistic Fuzzy Logics. Springer, Cham.

[8] Atanassov, K. (2017) New intuitionistic fuzzy extended modal operators. Notes on Intuitionistic Fuzzy Sets, 23(4), 40-45.

[9] Atanassov, K. (2018) Two intuitionistic fuzzy modallevel operators. In:- Advances in Fuzzy Logic and technology 2017, Springer, Cham, Vol. 1, 85-98.

[10] Atanassov, K., \& Gargov, G. (1989) Interval-valued intuitionistic fuzzy sets. Fuzzy Sets and Systems, 31(3), 343-349.

[11] Kaufmann, A. (1977) Introduction a la Theorie des Sour-Ensembles Flous, Paris, Masson. 